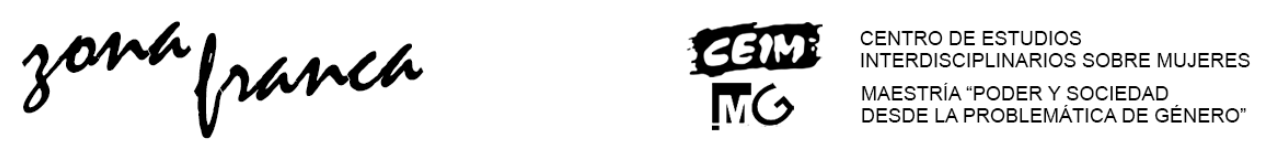

\title{
Aborto legal seguro y gratuito. Entre la legalidad y la clandestinidad. El caso del Hospital Dr. Roque Sáenz Peña de la ciudad de Rosario.
}

Miranda Ferrara

\section{Resumen}

Este artículo se basa en una tesina de $\operatorname{grado}^{1}$ que hace referencia a las políticas de salud de la mujer. Tiene como objetivo informar acerca del dispositivo institucional de Consejerías y más específicamente centrarse en la Consejería en salud sexual y reproductiva del Hospital Dr. Roque Sáenz Peña de la ciudad de Rosario, seleccionada por la excepcionalidad en la implementación de esa política. A su vez, se busca visibilizar el rol de acompañamiento del Socorrismo como una estrategia no institucional que se articula con el sistema de salud público para garantizar la libre decisión sobre el cuerpo.

Palabras claves: Salud sexual y reproductiva - aborto - RosarioConsejerías - Socorrismo

\section{Safe, legal and free abortion. Between legality and secrecy. The case of the Dr. Roque Sáenz Peña Hospital in the city of Rosario.}

\section{Abstract}

This article is based on a thesis that refers to women's health policies. Its objective is to inform about the institutional device of Counseling and more specifically to focus on the Counseling in sexual and reproductive health of the Dr. Roque Sáenz Peña Hospital in the city of Rosario, selected for the exceptionality in the implementation of that policy. At the same time, we seek to visualize the role of accompaniment of Socorrismo as a non-institutional strategy that articulates with the public health system to guarantee free decision about the body.

Keywords: Sexual and reproductive health - abortion - Rosario - Counseling - Socorrismo

*Universidad Nacional de Rosario. Contacto: miri ferrara67@hotmail.com

Ferrara, Miranda. "Aborto legal seguro y gratuito. Entre la legalidad y la clandestinidad. El caso del Hospital Dr. Roque Sáenz Peña de la ciudad de Rosario" en Zona Franca. Revista del Centro de estudios Interdisciplinario sobre las Mujeres, y de la Maestría poder y sociedad desde la problemática de Género, №26, 2018 pp.52-79. ISSN, 2545-6504 Recibido: 26 de mayo, 2018; Aceptado: 26 de septiembre 2018

Revista Zona Franca- Centro de estudios interdisciplinario sobre las mujeres (CEIM)- Maestría poder y sociedad desde la problemática de género (MG), Rosario, Argentina. ISSN, 2545-6504 http://zonafranca.unr.edu.ar/index.php/ZonaFranca| Numero 26 (2018). 


\section{Introducción}

Con todos los pronósticos en contra, con un gobierno conservador, con un Papa argentino y luego de presentarse por séptima vez, el año 2018 se erigió como el momento histórico en el cual se debatió por primera vez en el Congreso de la Nación argentina el proyecto que propone la legalización del aborto. Si bien este proyecto no fue aprobado, por el momento, lejos de considerarse una derrota, brindó la posibilidad de conocer a aquellos que nos representan y qué intereses encarnan y principalmente cristalizó el trabajo articulado del movimiento de mujeres y grupos feministas que luchan en pos de adquirir más y mejores derechos.

Históricamente, los derechos sexuales y reproductivos fueron tenidos en cuenta en base a una asociación directa entre mujer y maternidad, en donde el foco sobre la salud de la mujer estaba ligado al proceso reproductivo como factor de producción. Esta concepción que si bien continúa vigente, fue perdiendo peso paulatinamente entendiendo la importancia de los servicios de salud, del derecho al goce y de la libre expresión sexual. Es decir que "la deconstrucción de la historia de la vida privada fue un camino para que las mujeres comprendieran las formas de poder que eran ejercidas sobre sus cuerpos y que los transformaban en un lugar de desposeimiento de la propia existencia". (Ávila, M. B. 1999, p.64)

La desigualdad de género y la discriminación han sido y continúan siendo aún hoy, grandes obstáculos que deben ser enfrentados vehementemente. Los Derechos sexuales y reproductivos "fueron creados y propuestos por los 
movimientos de mujeres para hacer más justa y democrática la vida social y extender la idea de igualdad y libertad de la esfera pública a la esfera privada. Deben ser ejercidos sin ningún tipo de coerción o discriminación, porque son parte del ejercicio de la ciudadanía" ${ }^{2}$. Al ser la salud un derecho humano esencial, los derechos sexuales y reproductivos son inalienables y forman parte integral de los mismos. Entendiendo a la salud como un fenómeno que trasciende el campo de la medicina, de lo meramente biológico y en la cual se hace necesaria la interdisciplinariedad para alcanzar una mirada más integral y poder comprender sus múltiples dimensiones.

Es por este motivo que los estudios de las políticas públicas están estrechamente conectados con la relación entre el Estado y la sociedad. Esta relación no ha sido nunca estática sino que se ha ido transformando con el devenir de la historia. Es decir que "la política pública cristaliza una relación social, resultado de la interacción estado-sociedad en cada momento histórico" (Oszlak y O'Donnell, 1981; Barukel, 2014).

No es objeto de este trabajo dilucidar las tensiones de esta complicada relación, sin embargo consideramos que es un buen punto de partida para el estudio de la implementación de las políticas públicas, es decir "ese espacio intermedio del actuar, el conjunto de acciones que transformó las intenciones en resultados observables" (Aguilar Villanueva, L., 1993, p. 44).

${ }^{2}$ Carta de Guanabara. Diciembre 2001. Recuperado de: http://agendadelasmujeres.com.ar/notadesplegada.php?id=2465

Revista Zona Franca- Centro de estudios interdisciplinario sobre las mujeres (CEIM)- Maestría poder y sociedad desde la problemática de género (MG), Rosario, Argentina. ISSN, 2545-6504 http://zonafranca.unr.edu.ar/index.php/ZonaFranca| Numero 26 (2018). 
De esta manera, en un primer apartado se analizará la política de salud sexual y reproductiva referida a la creación de las Consejerías en Salud Sexual y Reproductiva a nivel nacional realizando un recorrido histórico del caso particular del Hospital Dr. Roque Sáenz Peña de la ciudad de Rosario (de ahora en más HRSP), considerado de vanguardia por darle cauce a demandas sociales previo a la existencia de legislación alguna. Es de sumo interés investigar y dar a conocer las herramientas de consejería dado que poseen una corta vida institucional lo cual motivó la utilización de una metodología eminentemente cualitativa con un gran apoyo en entrevistas, documentos y periódicos.

Se estudia la implementación de la Consejería del Hospital Dr. Roque Sáenz Peña desde el modelo "bottom up" en donde se toma una perspectiva de análisis desde abajo hacia arriba. Es decir, que se les da una gran importancia a Ixs operadorxs de la política, a aquellxs que la hacen efectivamente posible y que mantienen un contacto directo con Ixs ciudadanxs y conocen el territorio donde ejecutarla. Este modelo enfatiza la multiplicidad de actorxs intervinientes en las políticas públicas, reconociendo no solo aquellxs que participan de primera mano, sino también aquellos grupos de presión que manifiestan interés en los resultados de la aplicación de una política. Esta perspectiva es sumamente adecuada ya que nos brinda un estudio y análisis más acabado de la política en cuestión y el cual resulta de gran valor para poder reconocer las acciones diseñadas, comprendiendo que la implementación es un proceso constante, que se renueva constantemente a raíz del surgimiento de diversos desafíos alimentados por Ixs 
Aborto legal seguro y gratuito. Entre la legalidad y la clandestinidad. El caso del Hospital Dr.

Roque Sáenz Peña de la ciudad de Rosario.

usuarixs y Ixs trabajadorxs del Hospital que son Ixs implementadorxs de esta política.

A raíz de la inexistencia de registros formales fundados en la ilegalidad en la que se inició la Consejería del HRSP, no se ha podido definir el trabajo dentro de un período determinado. Sin embargo, y para reconstruir este proceso, este artículo se respalda a partir del discurso oral de diversxs trabajadorxs de la salud del Hospital Dr. Roque Sáenz Peña que fueron entrevistadxs ${ }^{3}$ y tomadxs como unidad de análisis.

En un segundo momento, se detallará acerca de la acción de las Socorristas en Red como una estrategia no institucional producto del activismo feminista que circula entre mujeres que desean interrumpir el embarazo, entendiendo que la legalidad del aborto trasciende las leyes, para luego llegar a las reflexiones finales.

\section{Derecho al aborto}

La legislación de nuestro país es restrictiva y penalizadora con respecto al aborto a raíz de ser considerado ilegal y de erigirse bajo el mandato de la mujer madre. Se encuentra expresado en el Código Penal Artículo 85 como un delito contra las personas que pena a quien lo efectúa y a la mujer que se causara o consintiera dicha práctica. El aborto es una práctica considerada ilegal, pero lejos de dejar de practicarse, continúan realizándose de manera insegura y clandestina

\footnotetext{
${ }^{3}$ Lxs entrevistadxs son parte del Anexo de la Tesina de Grado para obtener la Licenciatura en Ciencia Política de la Universidad Nacional de Rosario, Santa Fe, Argentina.
}

Revista Zona Franca- Centro de estudios interdisciplinario sobre las mujeres (CEIM)- Maestría poder y sociedad desde la problemática de género (MG), Rosario, Argentina. ISSN, 2545-6504 http://zonafranca.unr.edu.ar/index.php/ZonaFranca| Numero 26 (2018). 
poniendo en riesgo la vida mujeres que no poseen acceso a condiciones básicas de salud. El acceso al aborto es una problemática de clase, de justicia social y de salud pública que implica grandes desigualdades. A su vez, en el artículo № 86 se expresa que el aborto no es punible cuando se practica por un/a médicx diplomadx con el consentimiento de la mujer encinta en dos situaciones, por un lado, "si se ha hecho con el fin de evitar un peligro para la vida o la salud de la madre y si este peligro no puede ser evitado por otros medios" ${ }^{4}$ "si el embarazo proviene de una violación o de un atentado al pudor cometido sobre una mujer idiota o demente. En este caso, el consentimiento de su representante legal deberá ser requerido para el aborto." ${ }^{5}$ Sin embargo, en Marzo de 2012, la Suprema Corte de Justicia argentina precisó que el aborto es no punible en violaciones cometidas sobre cualquier mujer y además indicó que no es necesario recurrir a la justicia para su realización. No obstante, "en los hechos, no hay aborto accesible en el sistema de salud, aun en los casos no punibles. Los abortos son siempre hechos, con diferentes condiciones de costos, seguridad y eficacia, de manera clandestina." (Pecheny, M. y Petracci, M. 2006, p. 59).

Diversas investigaciones coinciden en plantear que el aborto inseguro es la primera causa directa de mortalidad de mujeres gestantes jóvenes y de escasos recursos. Solo para tener una referencia, al iniciar el año 2015 el entonces Ministro de Salud Daniel Gollán habló abiertamente sobre aborto y expresó que se calculan

\footnotetext{
${ }^{4}$ Argentina. Código Penal. Artículo № 86. Recuperado de: http://servicios.infoleg.gob.ar/infoleglnternet/anexos/15000-19999/16546/texact.htm\#15

${ }^{5}$ ibídem

Revista Zona Franca- Centro de estudios interdisciplinario sobre las mujeres (CEIM)- Maestría poder y sociedad desde la problemática de género (MG), Rosario, Argentina. ISSN, 2545-6504 http://zonafranca.unr.edu.ar/index.php/ZonaFranca| Numero 26 (2018). 
Aborto legal seguro y gratuito. Entre la legalidad y la clandestinidad. El caso del Hospital Dr.

Roque Sáenz Peña de la ciudad de Rosario.

en Argentina 500 mil abortos ${ }^{6}$ anualmente y que según datos oficiales disponibles del 2013, en ese mismo año 245 mujeres murieron por causas relacionadas con el embarazo y que de las mismas, 50 mujeres murieron por abortos ${ }^{7}$. La importancia de la despenalización total del aborto es de gran magnitud a raíz de los beneficios para la salud de las mujeres en cuanto a la disminución de la mortalidad, siendo que la clandestinidad de esta práctica implica la principal causa de muerte de personas gestantes.

A partir del año 2015 se hace expresa una modificación fundamental en el relanzamiento del Protocolo para la atención integral de las personas con interrupción legal del embarazo, donde hay un reconocimiento explícito del derecho a decidir, teniendo además resolución ministerial y expandiéndose a nivel nacional. A su vez, se incorporan distintas dimensiones para solicitar una interrupción legal del embarazo las cuales pueden ser: "el embarazo representa un peligro para la vida de la mujer y este peligro no pueda ser evitado por otros medios; el embarazo representa un peligro para la salud de la mujer y este peligro no pueda ser evitado por otros medios; el embarazo proviene de una violación; el

\footnotetext{
${ }^{6}$ Vale mencionar que es un número aproximado a raíz de que al ser una práctica clandestina no se disponen de datos precisos. La información disponible se refiere a hospitalizaciones producidas en efectores públicos por complicaciones relacionadas con abortos, en los cuales no se distingue entre abortos espontáneos y provocados, por lo que representan solo una fracción de los que ocurren anualmente.

${ }^{7}$ Argentina. Ministerio de Salud Presidencia de la Nación. (Diciembre de 2014) Estadísticas vitales. Año 2013. Información Básica. Serie 5. Número 57. Recuperado de: http://www.deis.msal.gov.ar/Publicaciones/Archivos/Serie5Nro57.pdf

Revista Zona Franca- Centro de estudios interdisciplinario sobre las mujeres (CEIM)- Maestría poder y sociedad desde la problemática de género (MG), Rosario, Argentina. ISSN, 2545-6504 http://zonafranca.unr.edu.ar/index.php/ZonaFranca| Numero 26 (2018). 
embarazo proviene de una violación sobre una mujer con discapacidad intelectual o mental." Retomamos a la autora Silvia Levín (2013) quien plantea que:

Las relaciones de género, que operan en el orden político, socioeconómico y cultural, son el resultado de varios sistemas de opresión -de sexo, de clase, de etnia, etc.que funcionan de manera "entrecruzada" y adquieren un carácter estructural en la sociedad. El sistema androcéntrico que rige esas relaciones somete a las mujeres a un mundo ideal en el cual los patrones que norman la vida social son masculinos, de dominación, jerárquicos y de poder. (p. 13)

Cabe preguntarse si el sistema político de un Estado de Derecho democrático puede obligar a las mujeres a entregar sus propias vidas para preservar otras. Al no legalizarse el aborto se vulneran derechos, primordialmente el derecho a la vida dado que el aborto ilegal genera complicaciones que llevan a muertes evitables.

A su vez, es de tener en cuenta la importancia de la legalización del aborto a raíz de la resistencia que presenta el personal médico vulnerando así el derecho de las personas gestantes. La no existencia del derecho al aborto y su consecuente penalización no es más que la intromisión gubernamental en el cuerpo de las mujeres, siendo una enorme deuda de la democracia que nos interpela a todxs como sociedad.

\footnotetext{
${ }^{8}$ Argentina. Ministerio de Salud. (2015). Protocolo para la atención integral de las personas con derecho a la interrupción legal del embarazo. Programa Nacional de Salud Sexual y Procreación Responsable.

Revista Zona Franca- Centro de estudios interdisciplinario sobre las mujeres (CEIM)- Maestría poder y sociedad desde la problemática de género (MG), Rosario, Argentina. ISSN, 2545-6504 http://zonafranca.unr.edu.ar/index.php/ZonaFrancal Numero 26 (2018). 
Aborto legal seguro y gratuito. Entre la legalidad y la clandestinidad. El caso del Hospital Dr.

Roque Sáenz Peña de la ciudad de Rosario.

\section{Consejerías en Salud Sexual y Reproductiva}

Las "consejerías" se encuentran en el marco de la normativa vigente en nuestro país, considerándose como ley fundante aquella que alude al Programa Nacional de Salud Sexual y Procreación Responsable sancionada en el año 2003. Se parte desde la base de la inclusión de los derechos sexuales y reproductivos como parte integral de los derechos humanos ubicados en los principales tratados Internacionales con rango constitucional reconocidos en la reforma de la Constitución Nacional de 1994. En el año 2010 a nivel Nacional se presentó un documento de trabajo que expresó una propuesta de diseño, organización e implementación de Consejerías en salud sexual y salud reproductiva como incentivo a la creación del dispositivo brindando información para su implementación. El documento de trabajo se ha ido actualizando dando lugar en 2014 a un nuevo escrito que fortalece los espacios de consejerías integrales y ofrece diversas herramientas para organizar y gestionar estos espacios en pos de ejercer los derechos sexuales y reproductivos. La implementación de las Consejerías hace referencia a una variación en el modelo tradicional ${ }^{9}$ de atención de la salud centrándose en la autonomía e información de Ixs usuarixs en lo que respecta a la sexualidad y cuidado del cuerpo como en la función reproductiva. Al

${ }^{9}$ El modelo de atención tradicional en las instituciones de salud se centra en el aspecto biológico. El acto médico, o la consulta, repite un mismo modelo que organiza y estructura la atención que cada profesional aprendió y fue construyendo a lo largo de su experiencia. Ese aprendizaje es sostenido, en la gran mayoría de los casos, desde la construcción del pensamiento clínico básicamente hipotético-deductivo. Se aísla la dimensión subjetiva en pos de lograr la objetividad deseada del proceso clínico.

Revista Zona Franca- Centro de estudios interdisciplinario sobre las mujeres (CEIM)- Maestría poder y sociedad desde la problemática de género (MG), Rosario, Argentina. ISSN, 2545-6504 http://zonafranca.unr.edu.ar/index.php/ZonaFranca| Numero 26 (2018). 
contrario de lo que podría indicar el nombre "Consejería", no se pretende proyectar la idea de aconsejar, sino de empoderar a Ixs usuarixs mediante la escucha y la información para la toma de decisiones autónomas.

De esta manera se tienen en cuenta las particularidades presentes en cada caso, la diversidad sexual, las condiciones de vida y socio culturales de la población usuaria. Se propone como objetivo de base fortalecer la autonomía para la toma de decisiones sobre la salud sexual y reproductiva de manera libre, consciente e informada. Asimismo, la consejería se erige como un espacio de promoción, prevención y asistencia como parte integrada al proceso de atención de la salud aproximando a Ixs usuarixs a sus derechos. Se propone acompañar en lo que respecta a la toma de decisiones para la construcción de autonomía acerca de anticoncepción, embarazo, parto, interrupción del embarazo (pre y post aborto), ejercicio de la sexualidad, prácticas de cuidado, identidad sexual y de género entre otras.

Es necesaria una conjugación de factores y que los mismos se mantengan a lo largo del tiempo, hay negociaciones y diversos obstáculos que superar para la creación de Consejerías, y como veremos, el caso de la ciudad de Rosario no es la excepción.

\section{Salud en la ciudad de Rosario}

En nuestra ciudad si bien desde los '80 se le comienza a dar relevancia a las temáticas relacionadas con la autonomía y los derechos de la mujer, se pueden considerar dos momentos de suma importancia. Por un lado y a partir de un proyecto presentado por el movimiento feminista nace en 2007 la ordenanza 
Aborto legal seguro y gratuito. Entre la legalidad y la clandestinidad. El caso del Hospital Dr.

Roque Sáenz Peña de la ciudad de Rosario.

que regula el primer protocolo de aborto no punible del país siendo de vanguardia al contemplar el aborto en caso de violación, peligro para la vida o la salud de la madre. Y por otro lado, a fines del 2014 cuando se aprueba la creación de las Consejerías Integrales en Salud Sexual y Reproductiva (CISSyR) ${ }^{10}$ como dispositivos encargados de promover y prevenir la salud sexual y reproductiva con perspectiva de derechos y género. Estas Consejerías están conformadas por un equipo de trabajo integral e interdisciplinario (psicología, psicología social, trabajo social, sociología, antropología, derecho, educación, etc.). A su vez, tienen como meta principal brindar información de manera sencilla, entregar anticonceptivos mediante una atención privada y personalizada con el fin último de impulsar el empoderamiento para la toma de decisiones para tener una vida sexual saludable y un conocimiento claro acerca de los derechos sexuales y reproductivos.

Vale remarcar que la legislación existente a nivel municipal, provincial, nacional e internacional no ha seguido un orden específico, sino que se ha ido desarrollando de manera muy heterogénea.

En lo que respecta al sistema de Salud Pública de Rosario ${ }^{11}$ se trabaja en red y se divide en tres niveles. El primer nivel constituye el espacio de mayor resolución y está conformado por los Centros de Salud Municipales (actualmente y según datos oficiales publicados en la página de la Municipalidad de Rosario, existen 50 centros de salud) y algunas vecinales, distribuidas estratégicamente en

10 Municipalidad de Rosario. Ordenanza № 9310. Recuperado de Boletín Oficial Electrónico: http://www.rosario.gov.ar/normativa/verArchivo?tipo=pdf\&id=102599
${ }^{11}$ Página
Oficial
Municipalidad
de
Rosario.
Recuperado
de: http://www.rosario.gov.ar/sitio/salud/sistemasalud1.jsp

Revista Zona Franca- Centro de estudios interdisciplinario sobre las mujeres (CEIM)- Maestría poder y sociedad desde la problemática de género (MG), Rosario, Argentina. ISSN, 2545-6504 http://zonafranca.unr.edu.ar/index.php/ZonaFranca| Numero 26 (2018). 
todo el municipio y organizados distritalmente (actualmente son seis). Es en los centros de salud donde se da el "primer nivel de contacto de los individuos, la familia y la comunidad con el sistema nacional de salud, llevando lo más cerca posible la atención de salud al lugar donde residen y trabajan las personas, y constituye el primer elemento de un proceso permanente de asistencia sanitaria."12 El segundo nivel de atención está constituido por tres hospitales: "Dr. Roque Sáenz Peña”, "Intendente Gabriel Carrasco" y “Juan Bautista Alberdi”, un servicio de Internación domiciliaria pediátrica y de adultos, dos maternidades, un Instituto de Rehabilitación (ILAR) y el Centro de Especialidades Médicas Ambulatorias de Rosario (CEMAR). Este nivel está destinado a la atención de consultas especializadas, con un desarrollo importante de exámenes complementarios y cirugía ambulatoria priorizando un tratamiento inmediato. Por último, el tercer nivel está constituido por las áreas de mayor tecnología y alta complejidad con el Hospital de Niños "Víctor J. Vilela" y el Hospital de Emergencias "Dr. Clemente Álvarez" (HECA). Esta red se completa con la distribución gratuita de medicamentos y el Servicio Integrado de Emergencias Sanitarias (SIES), con ambulancias para traslados y emergencias.

\section{Historia de la Consejería en Salud Sexual y Reproductiva del HRSP}

El HRSP está ubicado en la zona sur de la ciudad de Rosario y entendiendo las redes de salud municipales, coordina la atención sanitaria con los Centros de Atención Primaria cercanos.

\footnotetext{
${ }^{12}$ Declaración de Alma Ata $1978 . \quad$ Disponible en: http://www.promocion.salud.gob.mx/dgps/descargas1/promocion/1 declaracion deALMA ATA. pdf
}

Revista Zona Franca- Centro de estudios interdisciplinario sobre las mujeres (CEIM)- Maestría poder y sociedad desde la problemática de género (MG), Rosario, Argentina. ISSN, 2545-6504 http://zonafranca.unr.edu.ar/index.php/ZonaFranca| Numero 26 (2018). 
Para reconstruir la historia del Hospital Dr. Roque Sáenz Peña se utilizaron como fuentes primarias y como técnica de recolección de datos principalmente entrevistas en profundidad realizadas a trabajadorxs de la salud, especialmente de la Consejería en Salud Sexual y reproductiva y a diversxs actorxs relevantes para el estudio de la política en cuestión.

Se realizó una entrevista a Daniel Teppaz, que ha sido Director del HRSP, ha realizado su residencia médica y colaborado en la Consejería de dicho Hospital conociendo de primera mano el funcionamiento interno del efector y pudiendo brindar una visión más integral. A su vez, ha estado involucrado desde un primer momento con el origen del primer protocolo de aborto no punible en el país que surge en la ciudad de Rosario. En cuanto a las trabajadoras de la Consejería, se entrevistó a Susana Arminchiardi, ex Jefa del área de Trabajo Social del HRSP, contando una gran trayectoria en la Consejería y que continúa involucrada en la temática, y Silvia Totó, Jefa del área de Ginecología quien colaboró en la reconstrucción de la historia de la Consejería. Asimismo, se entrevistó a Mabel Gabarra, una referente histórica por el derecho a decidir y una de las primeras mujeres en dar vida a la Campaña Nacional por el Derecho al Aborto, legal, seguro y gratuito que es la idea máxima a partir de la cual se engloba el presente trabajo y que colaboró en el registro de la historización de la política en la ciudad de Rosario.

Por último, pero no menos importante, se incorporó la palabra de Marina Acuña de Socorristas en Red, quien explicó el funcionamiento de esta 
organización y comprendiendo la manera de accionar en relación con el servicio público de salud de la ciudad de Rosario.

Además se revisaron recortes de diario, diversos documentos de trabajo, protocolos, leyes nacionales, provinciales y municipales que han sido de gran utilidad para comprender de manera integral la política en estudio.

Para comprender la excepcionalidad de la Consejería del HRSP, nos remontamos a varias décadas atrás. La Consejería del efector es pionera en materia de derechos sexuales y reproductivos, iniciándose desde los años `80. Para contextualizar, durante la dictadura militar estaban prohibidos los consultorios de salud sexual y anticoncepción en los efectores públicos, sin embargo en el HRSP en los inicios de 1980, se materializó un consultorio de anticoncepción que era realizado por una médica (de la cual no hemos conseguido su nombre), quien lo llevaba adelante, en palabras de la Jefa del área de Ginecología Silvia Totó, de manera "muy artesanal". Daniel Teppaz menciona en la entrevista realizada que el Hospital utilizaba métodos anticonceptivos y se realizaban cirugías anticonceptivas pero siempre con mucho sigilo a raíz del contexto de prohibición que acabamos de mencionar.

Para la segunda mitad de la década del ` 80 se creó un grupo interdisciplinario de atención al adolescente integrado por una trabajadora social, una médica obstetra, una ginecóloga y una psicóloga. Este antecedente es de gran importancia a raíz de que demuestra la colaboración existente entre Ixs trabajadorxs dado que estas temáticas eran tratadas solo a puertas cerradas en el consultorio y de manera independiente. 
Un cambio notable se evidencia con la llegada de la Democracia y con la derogación del decreto №659 de 1974 que prohibía las actividades relacionadas con el control de la natalidad, lo cual dio rienda suelta al libre establecimiento del consultorio de anticoncepción. Vale aclarar que en la Facultad de Medicina no se enseñaban métodos anticonceptivos y sin embargo las prácticas médicas realizadas en el HRSP incluían esta formación.

Lxs entrevistadxs manifestaron que cuando las mujeres se acercaban al efector por complicaciones por abortos clandestinos o ilegales la institución se caracterizó por no denunciarlas, aun cuando existían disposiciones que planteaban lo contrario, lo que significaba que las mujeres ante el temor de ser denunciadas, "demoraran más en ir a los hospitales y llegaran más graves aún." (Vallina, C. y Monje, id).

Tal como venimos observando, el HRSP fue pionero al funcionar en un primer momento como consultorio de anticoncepción y luego con el nuevo milenio surge una Consejería sin legislación fundante ni claridad con respecto a su funcionamiento, lo cual estuvo relacionado con la existencia de una multiplicidad de factores relacionadas con la puesta en escena del aborto y la anticoncepción y de demandas que no encontraban su cauce.

Vale aclarar que la creación de la Consejería no se dio de manera lineal como lo estamos planteando, sino que como sostuvo la ex Jefa del área de Trabajo Social Susana Arminchiardi, fue producto de un proceso que comenzó en el año 2003 a partir del trabajo interdisciplinario y articulado entre las áreas de Ginecología, Salud mental, Maternidad y Trabajo social en donde se comenzó a 
trabajar con los recursos disponibles y se comenzaron a coordinaron esfuerzos para crear este espacio que carecía de antecedentes. De esta manera, se puede considerar como año fundacional de la Consejería en Salud Sexual y Reproductiva en el 2013.

\section{Funcionamiento de la Consejería del HRSP}

El Hospital Dr. Roque Sáenz Peña es un efector donde históricamente se ha trabajado con la anticoncepción, pero no sobre los derechos de la mujer. A pesar de los grandes avances logrados, Ixs entrevistadxs manifiestan que aún hoy diversxs profesionales interfieren en el derecho de la mujer a raíz de jerarquizar ideales y deseos propios, sin considerar a Ixs usuarixs como sujetxs de derecho.

De esta manera y a partir de luchas cotidianas, se comenzó a trabajar y garantizar desde la aprobación de Ixs Directorxs y Jefxs de diversas áreas del Hospital hasta lograr la presencia de trabajadorxs de la salud que no sean objetorxs de conciencia para poder brindar un servicio acorde a las necesidades de Ixs usuarixs. A su vez, es necesario el apoyo del sector de enfermería, cirujanxs y demás profesionales que implican garantizar una estructura, siendo un trabajo arduo diario y en constante construcción llevada adelante por Ixs mismos trabajadorxs de la salud.

La Consejería que actualmente posee el Hospital es y ha sido fruto de lucha de Ixs trabajadorxs de la Consejería que han conseguido (y continúan haciéndolo) crear un espacio de fácil acceso, abierto y amigable, en donde se construye un vínculo de compromiso y aprendizaje mutuo y que no genere incomodidad a las personas usuarias, que sea privado y en consecuencia, adquirir una estabilidad 
Aborto legal seguro y gratuito. Entre la legalidad y la clandestinidad. El caso del Hospital Dr.

Roque Sáenz Peña de la ciudad de Rosario.

para referenciarse tanto hacia adentro de la comunidad hospitalaria como hacia fuera para la población en general.

La posibilidad de llevar adelante la Consejería desde el espacio y tiempos de atención, fue una herramienta que ordenó el trabajo de Ixs profesionales y de las usuarias que anteriormente se presentaban en el Hospital sin contención y sin áreas específicas donde canalizar sus necesidades.

La Consejería recibe una multiplicidad de consultas relacionadas con anticoncepción y controles médicos, pero la mayor demanda es acerca de la interrupción legal del embarazo donde confluyen usuarixs de diferentes edades, diferentes niveles de apropiación de los derechos sexuales y reproductivos, con obra social o sin, acompañadxs por algún familiar, por sus parejas o solxs.

Allí se trabaja la interrupción legal del embarazo concretamente hasta las 12 semanas y se ofrece hasta el momento una única manera medicamentosa mediante el uso del misoprostol ${ }^{13}$. El hecho de poder darle la posibilidad a Ixs usuarixs de elegir de qué manera realizar la interrupción es otro de los factores en los que actualmente se está trabajando, dado que para realizar una $\mathrm{AMEU}^{14}$ se requiere una movilización de recursos aún mayor lo cual es un poco más complicado pero no imposible. La existencia y circulación del misoprostol para

${ }^{13}$ El Misoprostol es un nuevo análogo de Prostaglandina E1 (PG E1) que comenzó a ser vendido en las farmacias de América Latina desde fines de la década de 1980 con el nombre comercial de Cytotec $\AA$, como tratamiento de la úlcera péptica, especialmente en los casos provocados por el uso de anti-inflamatorios no esferoidales 5-7. Su uso con ese fin está contraindicado en embarazadas porque podría causar un aborto.

${ }^{14}$ La aspiración manual endouterina (AMEU) es un método seguro y eficaz para la interrupción del embarazo, que consiste en la evacuación del contenido del útero mediante el uso de un aspirador manual

Revista Zona Franca- Centro de estudios interdisciplinario sobre las mujeres (CEIM)- Maestría poder y sociedad desde la problemática de género (MG), Rosario, Argentina. ISSN, 2545-6504 http://zonafranca.unr.edu.ar/index.php/ZonaFranca| Numero 26 (2018).

Página 68 
interrumpir el embarazo, redujo visiblemente el hecho de que las mujeres que decidan abortar lo hagan utilizando métodos inseguros. En promedio asisten a la Consejería en Salud Sexual y Reproductiva del HRSP entre 6 y 8 mujeres semanalmente en busca de asesoramiento por interrupción legal del embarazo en donde los causales salud y social tienen mucho peso. Lxs trabajadorxs de la salud también se proponen realizar un acompañamiento en todo el proceso en donde se contemple la realización de ecografías para verificar que la interrupción haya sido exitosa. El objetivo es trabajar en un espacio de post-aborto en donde se brinde información adecuada acerca de la importancia de cuidar la salud y también de brindar información sobre los derechos sexuales y reproductivos.

A su vez, es importante mencionar que el HRSP no recibe solo usuarixs de los Distritos Sur y Sudoeste de la ciudad de Rosario, sino que también reciben usuarixs de diversos puntos de la ciudad y de otras localidades o provincias cercanas. Esta situación genera una saturación en el servicio, motivo por el cual Ixs trabajadorxs de la salud generan redes y colaboran con los centros de salud de la ciudad para que los mismos puedan llevar adelante interrupciones o derivar al Hospital más cercano y de referencia según al distrito al que pertenezcan entendiendo que de esta manera se brinda una mejor atención y más personalizada.

\section{Dificultades en el camino}

Si bien el objetivo de este trabajo radica en informar acerca de la existencia de Consejerías en Salud Sexual y Reproductiva y más específicamente visibilizar el caso del HRSP, también se entiende que más allá de la existencia de 
legislación, hay una gran resistencia en los Hospitales relacionados con la objeción de conciencia, de negarse a realizar anticoncepciones quirúrgicas, de no acompañar el proceso de interrupción legal del embarazo que implican enormes barreras para la atención de Ixs usuarixs y una gran frustración por no encontrar respuestas en servicios públicos ante derechos que deben ser cumplidos.

Según palabras de Ixs entrevistadxs, tanto en el HRSP que ocupa nuestro caso de estudio como en otras Consejerías, existen complicaciones que Ixs mismxs trabajadorxs de la salud deben negociar constantemente con otrxs profesionales para garantizar los servicios de Salud Sexual y Reproductiva y que de esta forma las demandas y derechos de Ixs usuarixs sean atendidas. Es de recalcar la importancia que posee el trabajo interdisciplinario y en equipo para lograr resultados y estabilidad en el tiempo, sin embargo las trabajadoras del HRSP manifiestan que es sumamente arduo que el resto de lxs compañerxs colaboren con el espacio.

A pesar de estos avances que mencionamos, es necesario un mayor compromiso por parte de Ixs trabajadorxs de la salud quienes por desconocimiento o de manera intencional retrasan y entorpecen la atención lo cual desemboca en la frustración y desamparo de Ixs usuarixs que ante la búsqueda de métodos inseguros para interrumpir el embarazo desembocan en la muerte.

La existencia de nuevas demandas y expectativas por parte de Ixs usuarixs supone un desafío de los equipos de salud en cuanto a sostener y ampliar la atención de la salud sexual y reproductiva. 
Cuando hablamos de reconocimiento planteamos que socialmente hablando, es conocida la existencia de la Consejería del HRSP como un espacio amigable, sin embargo dentro del Hospital no se la reconoce como tal debiendo lidiar con las decisiones políticas de Ixs gestorxs de la salud lo cual desemboca en que sean Ixs mismxs trabajadorxs quienes breguen por sostener este espacio.

A su vez y desde lo político institucional, Ixs mismxs trabajadorxs de la Consejería fundamentan que es necesario un sostén político y colaboración en la promoción por parte de la Municipalidad de Rosario que facilite la expansión y consolidación del trabajo que se realiza.

En cuanto al logro de ese reconocimiento, es necesaria una fuerte presencia en garantizar la aplicabilidad de la ley, es decir, es necesario que las autoridades se erijan como garantes al explicitar el amparo que la ley supone.

Estas cuestiones implican una modificación en el pensamiento y de patrones culturales producto de un proceso relacionado con el hecho de que las mujeres se adueñan cada vez más de sus derechos lo cual es producto de la lucha de los movimientos de mujeres y feministas.

Como hemos analizado, en la Consejería nada está agotado dado que es un espacio permeable a nuevas demandas y a partir de la escucha de las personas usuarias.

\section{Socorrismo}

Se considera necesario destacar la labor conjunta que existe entre la Consejería en Salud Sexual y Reproductiva del HRSP y las Socorristas en Red 
quienes desarrollaron una estrategia basada en la interrelación de estos espacios para tratar las interrupciones legales de embarazos.

Socorristas es una Red integrada por mujeres feministas que deciden involucrarse a partir de la práctica de "poner el cuerpo" tal como Marina Acuña, quien no es médica e integra la Red, lo expresa en la entrevista realizada. Esto significa la posibilidad de acompañar y luchar por la deuda histórica con las mujeres por parte del Estado. El acompañamiento no se realiza desde el ámbito medico profesional, sino que la organización están conformada por mujeres de diferentes trayectorias. Plantean la necesidad de la despenalización social que implica poner el aborto en debate y dejar de pensarlo como un tabú sino como una problemática de salud de las mujeres que debe ser atendida.

Las Socorristas brindan información segura sobre como abortar con misoprostol, reconociendo el mismo como el método más seguro y más efectivo para abortar hoy en la Argentina que se consigue de manera legal. Sin embargo, la entrevistada reconoce dificultades para conseguir el medicamento en las farmacias a raíz de diversos controles (doble receta, estampillados, DNI).

En nuestra ciudad las Socorristas tienen una relación con médicxs del sistema de salud, dentro de Ixs cuales se encuentran aquellxs del HRSP, con Ixs que trabajan de manera conjunta a partir de la utilización del protocolo de interrupción legal del embarazo. De esta manera, se establece una implementación más versátil reforzando la política de las Consejerías, y dando mejores respuestas a las mujeres que desean realizar una interrupción del 
embarazo garantizando de esta manera que se realice de manera segura y acompañada, tanto por las Socorristas como por Ixs médicxs.

Las Socorristas de nuestra ciudad reciben en promedio por semana entre 6 y 7 llamadas y se realiza el acompañamiento hasta la semana 12 del embarazo.

El socorrismo supone realizar un acompañamiento en todo el proceso de interrupción del embarazo. Se parte de un acompañamiento presencial, un encuentro grupal que se aborda con un equipo de socorristas y las mujeres que se comunican. Allí mediante una entrevista se aborda la situación particular de cada mujer, comprendiendo que cada mujer atraviesa la situación del aborto de manera distinta y al ser en grupo muchas veces se genera un lazo de apoyo con otrxs socorridxs. En un segundo momento y para obtener la receta, Ixs conectxn con médicxs amigables que atienden por obra social o son derivadxs a efectores de salud, como el caso particular de estudio. La entrevistada hace referencia a que la mayoría de los abortos se llevan adelante en el sistema de Salud Pública.

Una vez efectuada la interrupción se comunican telefónicamente para tener una entrevista postaborto en donde se solicita una ecografía de control para culminar con una última entrevista presencial en donde se socializa la experiencia.

Se entiende al aborto como una práctica que atraviesa al feminismo y que no implica poner en riesgo la salud de la mujer, se rescata el acompañamiento y solidaridad de las mujeres en un contexto patriarcal ${ }^{15}$.

${ }^{15}$ Es un sistema de dominación masculina que determina la opresión y subordinación de las mujeres poniendo énfasis en la sexualidad, no conformándose con las reivindicaciones logradas en el espacio público (como la igualdad en el trabajo, la educación o los derechos civiles y políticos) 
Aborto legal seguro y gratuito. Entre la legalidad y la clandestinidad. El caso del Hospital Dr. Roque Sáenz Peña de la ciudad de Rosario.

En este acompañamiento integral que realizan las socorristas, también tienen que luchar con ciertxs profesionales del sistema público que se niegan a realizar abortos o que indican de manera incorrecta cómo utilizar el misoprostol. De esta manera y a partir de visibilizaciones y de poner en agenda casos particulares se recurre a la denuncia, lo cual implica un antecedente más en la lucha dado que sienta precedentes para negociar con otrxs profesionales. La deuda pendiente sobre la lucha por la legalización del aborto hace referencia a la decisión misma de la mujer sobre su propio cuerpo, se basa en la libertad de la mujer de decidir cuándo ser madre o no.

Tal como pudimos ver, hay una gran multiplicidad de actores en la implementación de una política pública. La existencia de la Consejería del HRSP se materializa por la cristalización de demandas de mujeres que encuentran su cauce en Ixs trabajadorxes de la salud. La sanción de la ley del Programa de Salud Sexual y Reproductiva no implica per sé una política pública así como tampoco la existencia de la interrupción legal del embarazo en determinados causales implica el efectivo cumplimiento de sus excepciones. En el caso de la Consejería del HRSP, la política pública era anterior al surgimiento de la ley, lo cual no le quita importancia al sentar las bases sobre las cuales abordar la salud sexual y reproductiva y ordenar a Ixs implementadores de la política. Tal como vimos, la implementación no se da de manera lineal ni de una vez y para siempre, sino que se renueva constantemente y toma nuevos rumbos. Incorporar la práctica

sino que se hace necesario transformar el espacio privado, de allí surge el lema "lo personal, es político" (Varela, N. 2005, p. 85).

Revista Zona Franca- Centro de estudios interdisciplinario sobre las mujeres (CEIM)- Maestría poder y sociedad desde la problemática de género (MG), Rosario, Argentina. ISSN, 2545-6504 http://zonafranca.unr.edu.ar/index.php/ZonaFrancal Numero 26 (2018). 
del Socorrismo se refiere a correr la mirada de lo meramente institucional para comprender otras prácticas que se articulan con el sistema de salud público, generando lazos que funcionan y se sostienen para que las mujeres puedan decidir sobre su cuerpo. Esta práctica rompe con el saber médico profesional y supone un acompañamiento desde otra perspectiva y lucha por la garantía de salud tal como su concepción integral lo supone.

Es necesario garantizar el acceso universal a los servicios públicos de salud buscando justicia social y exigiendo al Estado su responsabilidad como garante del cumplimiento de la salud como derecho humano.

\section{Reflexiones finales}

Habiendo llegado casi a un final que no pretende ser cerrado, sino que tiene la intención de abrir nuevos interrogantes y nuevos caminos para ampliar derechos, se vislumbra que el trabajo realizado por Ixs profesionales de la Consejería del HRSP, como así lo hacen otrxs profesionales de distintos efectores públicos, es de una magnitud inconmensurable y en donde se lleva adelante una lucha constante.

En la realidad del efector coexisten ciertos aspectos que no hacen de la Consejería un dispositivo ideal lo cual da cuenta de un gran desgaste para Ixs profesionales que llevan adelante la práctica. En este sentido, se considera necesario repensar la estructura de los hospitales y centros de salud para que el dispositivo posea un espacio de jerarquía y un presupuesto que lo contemple. Es necesaria una mirada institucional más comprometida con la labor de lxs profesionales de la salud, garantizando el cumplimiento de leyes en el resguardo 
de las mujeres que solicitan atención médica. Consideramos que aquellxs profesionales que se niegan a realizar interrupciones legales de embarazos, no lo hacen desde el desconocimiento de sus facultades médicas legales, sino que son un reflejo del poder de control sobre el cuerpo de la mujer y por lo tanto de la libertad de decisión de las mismas. Esto se relaciona con el orden dominante que plantea el rol natural y obligatorio de mujer madre, en donde el derecho al aborto sugiere una modificación en la estructura, en ese orden patriarcal.

En este trabajo se ha podido tomar conocimiento de prácticas que están invisibilizadas en nuestra ciudad, tanto desde el socorrismo que es una práctica conocida por el boca en boca entre mujeres que desean abortar, así como también la existencia de Consejerías en distintos hospitales públicos que acogen a diversas mujeres ante la búsqueda común de la libertad del cuerpo.

Se presentaron una conjugación de factores, desde lo jurídico y voluntades políticas, hasta las incansables luchas de los movimientos de mujeres y diversos sectores de la sociedad que acompañaron, como también a nivel social al lograrse cierta maduración que permitió la penetración del tema en la agenda de gobierno. Esto sienta las bases para alcanzar, entre otras, la deuda que se refiere a la conquista de la libertad del cuerpo de la mujer, lo que eliminaría la diferencia entre la vida y la muerte de las mujeres, es decir entre aquellas con recursos para ejercer su sexualidad y reproducción libremente y aquellas que deben hacerlo en la clandestinidad poniendo en riesgo su vida.

\section{Bibliografía}

Revista Zona Franca- Centro de estudios interdisciplinario sobre las mujeres (CEIM)- Maestría poder y sociedad desde la problemática de género (MG), Rosario, Argentina. ISSN, 2545-6504 http://zonafranca.unr.edu.ar/index.php/ZonaFranca| Numero 26 (2018). 
ÁVILA, María Betanía. (1999). Feminismo y ciudadanía: la producción de nuevos derechos en Scavone, L. (comp.). Género y salud reproductiva en América Latina. (pp. 5783). Libro Universitario Regional. Ed. Universidad de Costa Rica.

BARUKEL, Agustina. (2014). ¿Una nueva etapa en el estudio de políticas? Desafíos para la práctica teórica y la práctica profesional. En Díaz, C., Galano, N., Curti, G. (comps.). Miradas de políticas públicas. Como se enseña y aprende el análisis de políticas en América Latina. (pp. 197-224) Rosario: Editorial libros por demanda.

CARABAJAL, Mariana (26 de Abril de 2016). Un juicio para violar derechos. Página 12.

CRUZ VELANDIA, Israel (2005). La salud colectiva y la inclusión social de las personas con discapacidad. Investigación y Educación en Enfermería. 23(1), (pp. 92-101).

Recuperado

de:

http://www.scielo.org.co/scielo.php?script=sci arttext\&pid=S012053072005000100 $\underline{008}$

D'ATRI, Andrea (2011). Derecho al aborto, derechos sexuales y reproductivos en Argentina Sexo, mentiras y... silencio. Recuperado de: http://www.ips.org.ar/wpcontent/uploads/2011/03/Sexo-mentiras-y...-silencio.pdf

DE LOS RÍOS, Rebecca (1993). Género, salud y desarrollo un enfoque en construcción en Gómez Gómez E., De Los Ríos, R. Plaut R., Torres, C. y Yunes J. Género, mujer y salud en las Américas. (pp. 3-18) Publicación científica no541. Organización Panamericana de la Salud. WashingtonD.C.

FABBRI, Luciano (2013) "Apuntes sobre feminismos y construcción de poder popular". Colección en las calles y en las camas. Rosario. Puño y Letra. Editorialisimo de base.

FLACSO (2011). Modulo I. Determinantes sociales de la salud. El concepto de Salud. Evolución histórica. Acepciones actuales. (pp. 17-69). Recuperado de: http://salud.ciee.flacso.org.ar/files/flacso/Man Flacso/Modulo 1Parte1 Flac

so 2011.pdf

JIMÉNEZ, Carlos Andrés (Mayo - Agosto 2009). Innovaciones en la gestión local en salud: una aproximación desde el caso de la Municipalidad de Rosario en 
el período 1995-2000. Salud colectiva. 5(2). (pp. 211-224). Buenos Aires. Recuperado

de: http://www.scielo.org.ar/scielo.php?pid=S185182652009000200005\&script=sci art $\underline{\text { text }}$

LEVÍN, Silvia (5-8 Noviembre 2003). La Ciudadanía de la mujer en Argentina en el siglo XXI: las tensiones de un modelo cívico. VI Congreso Nacional de Ciencia Política. Sociedad Argentina de Análisis Político. Rosario.

(2013). Un nuevo criterio de justicia en Argentina: aborto por violación. La Aljaba. Segunda época. 17 (pp. 11-27). Recuperado de: http://www.scielo.org.ar/scielo.php?script=sci_arttext\&pid=S166957042013000100 001\#notas2

(2010) Derechos al revés: ¿salud sexual y salud reproductiva sin libertad? 1 ${ }^{\underline{a}}$ ed. Buenos Aires: Espacio Editorial.

LIBORIO, Mónica (2013) ¿Por qué hablar de salud colectiva? Cátedra de Medicina Preventiva y Social. Facultad de Ciencias Médicas. Universidad Nacional de Rosario. Revista Médica de Rosario. 79(3), pp. 136-141).

RAMOS, Silvina; BERGALLO, Paola; ROMERO, Mariana; ARIAS FEIJOO, Jimena (2009). El acceso al aborto permitido por la ley: un tema pendiente de la política de derechos humanos en Argentina en Derechos Humanos en Argentina (pp. 451-491, 1ㄹ ed.). Informe del Centro de Estudios Legales y Sociales (CELS). Buenos Aires: Siglo XXI Editores.

RAMOS, Silvana; GOGNA, Mónica; PETRACCI, Mónica; ROMERO, Mariana y SZULIK, Dalia (2001). Los médicos frente a la anticoncepción y el aborto: ¿Una transición ideológica? Buenos Aires: Centro de Estudios de Estado y Sociedad. Recuperado de: http://www.clacaidigital.info:8080/xmlui/bitstream/handle/123456789/77/Los $\mathrm{m}$ dicos.pdf?sequence $=1$

ROMERO, Mariana; ABALOS, Edgardo; RAMOS, Silvina (Marzo 2013). La situación de la mortalidad materna en Argentina y el Objetivo de Desarrollo del Milenio 5. Observatorio de Salud Sexual y Reproductiva (OSSyR). Número 8. 
SUBIRATS, Joan (1989). Análisis de políticas públicas y eficacia de la administración. INAP. (pp. 101-123; 125-138). Madrid.

TEPPAZ, Daniel (2007). Anticoncepción de emergencia en Derechos Sexuales y Reproductivos. Jornadas de Capacitación de Equipos de Salud. Conferencias y debates. $1^{1}$ ed. (pp. 43-49) Rosario: UNR Editora.

VALLINA, Cecilia y MONJE, Carolina (27 de Agosto de 2003). Bondesío ordena denunciar los casos de abortos terapéuticos. El Ciudadano, p.8.

VARELA, Nuria (2005). Feminismo para principiantes. Barcelona Ediciones. España.

VASALLO MANTILLA, Celestino (2007). Sexualidad: Salud Sexual. Prevención del VIH-SIDA. Revista Habanera de Ciencias Médicas. 6(Supl. 5). Recuperado de:

http://scielo.sld.cu/scielo.php?script=sci arttext\&pid=S1729519X2007000500014 